Backbeat Placement Affects Tempo Judgments

Bryn Hughes \& Dominique T. Vuvan

Under review at Psychomusicology as of May 26, 2020 
Backbeat Placement Affects Tempo Judgments

\author{
Bryn Hughes
}

University of Lethbridge, Canada

Dominique T. Vuvan

Skidmore College, United States 


\begin{abstract}
Research on tempo perception has shown that it is effectively modeled by tactus rate (musical pulse), and aligns with the theory of metrical hierarchy. This research typically draws from common-practice music (music from the Western European tradition, ca. 1750-1900), and therefore does not address traits found in other repertoire that may contend with these claims. The current study investigated the impact of the backbeat, a ubiquitous rhythmic feature of popular music, on tempo perception. The experiment asked listeners to compare the tempos of pairs of excerpts with the same tactus rate. Pairs of excerpts were always presented with different backbeats, shifting to either half-time or double-time. Results indicated that half-time trials were perceived to be slower, and double-time trials were perceived to be faster, despite identical tactus rates and metrical hierarchies. The findings provide empirical support for the idea that established theories of tempo perception and metrical hierarchy may not entirely extend to other musical styles, and that the backbeat may be a metrical feature of popular music, rather than simply a rhythmic one.
\end{abstract}

Keywords: Tempo Perception; Metrical Hierarchy; Backbeat; Popular Music 


\section{Backbeat Placement Affects Tempo Judgments}

In rock and jazz, it is relatively common for moments in the music to be referred to as "half time" or "double time." When the music is notated, this is sometimes even indicated on the score. These moments are intended to convey the sense that the tempo of the music has been halved or doubled, typically providing a stark sense of contrast with the surrounding music. Somewhat perplexing is that the effect is created with almost no change to the underlying musical structure. A simple halving or doubling of the number of backbeats creates a supposed sense of tempo shift for listeners and performers, a feeling that musical time is unfolding "half as fast" or "twice as fast," despite the underlying tactus and metrical hierarchy remaining constant. The half-time/double-time effect raises two interesting questions that motivated the experiments reported below: Does half time or double time actually represent a listener's perception of tempo, or is it simply a stylistic misnomer? Further, can a style-specific trait such as backbeat influence perception of the metrical hierarchy, and if so, how does this impact theories of meter and tempo that attempt to generalize across musical styles?

\section{Tempo Perception}

Tempo perception has been studied from numerous perspectives that will be helpful to review in defining precisely how our study fits into this line of research. To begin, we must tease apart the many different definitions of tempo that exist in the literature. Drake et al. (1999) differentiate musical and perceived tempo. Musical tempo refers to the tempo indicated on a score, which is in turn interpreted by performers. The tempo given typically refers to the tactus, which we consider to be the level of periodic beats (pulses), either present or implied in the music, to which most people entrain (Honing, 2013; London, 2004). Performers will produce this 
tempo to varying degrees of specificity; the outcome of this interpretation is referred to as performed musical tempo. In both cases, the tempo is easily calculated in terms of beats per minute (BPM). Performed tempo is perhaps the simplest measurement of tempo perception. Numerous studies have shown that beats-per-minute measurements are a relatively accurate account of musical speed (Jones \& Boltz, 1989; Moelants, 2006; Parncutt, 1994; Quinn \& Watt, 2006; van Noorden \& Moelants, 1999), and that participants' tapping behaviours (a kind of musical performance) typically reflect this pulse rate (Drake et al., 2000; P. A. Martens, 2011; McKinney \& Moelants, 2006; Snyder \& Krumhansl, 2001).

Perceived tempo is more difficult to calculate. One approach is to observe the inter onset interval (IOI), the duration between event onsets, typically measured in milliseconds. When listening to a single line of isochronous tones, the tempo is easily defined by the IOI. In music, however, this is much less clear due to the multiple layers of events that occur. Different parts of the music can unfold at different rates; even a single part can suggest multiple potential tactus rates. Listeners may attend to the tactus, and therefore the IOI between tactus points could serve as a measurement of tempo. However, listeners may attend to a different level of the metrical hierarchy and perceive its IOI as the defining tempo. For example, a musical line that is performed with quarter notes unfolding at 120 BPM may be perceived as "faster" than one at 200 BPM, because listeners may perceive the tactus of the second line to be at the half-note level (100 BPM) rather than the quarter-note level (200 BPM) of the metrical hierarchy (Martens \& Benadon, 2017). Moreover, a listener may even associate the mean number of events, or the event density, as a representation of tempo (Bisesi \& Vicario, 2015).

Further complicating matters is Jones and Boltz's theory of dynamic attending (1989) which suggests that, after attending to a referent level in the stimulus (typically the tactus), 
listeners will spend effort shifting their attention to events at different metrical levels. While spontaneous perceived tempo is often determined by the referent level, this rate may be consciously modified by listeners to reflect other metrical levels (Drake et al., 1999, pp. 192-3). Studies of tempo perception should be careful to stipulate whether stimuli are long enough for listeners to shift attention between metrical levels.

When asked to compare the speed of two isochronous sequences, participants are more sensitive to tempo changes with IOIs between 300 and 800 milliseconds. This is referred to as the zone of optimal processing (Drake \& Botte, 1993). Participants will produce a pulse within this range when spontaneously tapping with no musical input, or when asked to tap along to auditory sequences or musical sequences (Drake et al., 1999). When presented with random sequences, participants' tapping rates correspond to the mean IOI for each sequence. Further, Drake et al. found no significant difference between these results and studies that used isochronous sequences, suggesting that tapping rate may depend on the mean IOI as much as a fixed IOI, and that event density may have as great an impact on tempo perception as metric hierarchy (Drake et al., 1999). Ultimately, their findings suggest that perceived tempo is highly complex, and their findings show an influence of several factors, including fixed IOI, mean IOI, performed musical tempo, metric hierarchy, and event density.

Several efforts have been made to disentangle the influence of factors impacting tempo perception mentioned above. London (2011) asked participants to compare tempi for two musical stimuli; one with a faster tactus rate and one with a greater event density. He questioned whether metronome markings actually indicate beat rate, and whether beat rate is itself a measurement of perceived tempo. Furthermore, he noted that patterns with equivalent event density performed with the same metronome marking may project different metric organizations, 
which could cause the beat rate to be ambiguous and the passages to be perceived as having different tempi. Like Drake et al (1999), London posits that bodily synchronization may not correspond directly to tempo perception; and that tempo perception may reflect, as David Epstein suggests, a "sum of all factors within a piece" (1995, p. 99). In three experiments, London asked participants to compare the tempi of two musical patterns and found that metrical structure affected tempo judgments even in trials where the event density was equivalent between the two patterns. In situations where there was a mismatch between tactus and event density, the event density (specifically the presence or absence of the subdivision of the tactus) is more salient than the tactus. London concluded that differences in surface activity and metrical structure interfere with tempo judgments; event density is more salient than tactus rate when these parameters are incongruent; and that intentional focus or tapping does not improve accuracy in tempo judgements of this kind.

Further to the findings of Drake et al (1999) and London (2011), Boltz (2011) undertook a series of studies that investigated the impact of non-rhythmic musical elements, specifically pitch, timbre, and loudness on tempo perception. Boltz found that all of these factors significantly impacted participants' accuracy in a paired comparison task. Specifically, patterns that increased in pitch, brightness, and loudness across the duration of the pattern were perceived to be faster than those that maintained or decreased these factors, even when the BPM rate of these stimuli did not change.

The research discussed above provides substantial evidence for the idea that perceived tempo consists of something more than the "sum of its parts." All of the studies discussed thus far, though, deal specifically with stimuli drawn from, or presumed to cohere with, standard theories of common-practice rhythm and meter. While many scholars have investigated groove 
in popular music (Danielsen, 2010), relatively less work has been done on tempo perception, specifically. Snyder \& Krumhansl (2001) investigated pulse finding in ragtime piano music (which typically contains a strong backbeat); however, they did not study the impact of backbeat itself. Elowsson \& Frieberg (2013) developed a computational model for tempo perception that extracted specific features from audio, among which included IOIs between kick and snare drum onsets (isolating the backbeat) in a corpus of popular music. Interestingly, their model accounted for $90 \%$ of the variability in listener ratings of speed, taken from a previous experiment. The backbeat, specifically, accounted for a statistically significant portion of their explanation of the variance. While these findings are informative, they do not specifically isolate the impact of backbeat in a direct comparison task. We take this as our point of departure.

\section{The relation between meter and backbeat}

In traditional music theory, tactus is considered to be the level of periodic beats (pulses), either present or implied in the music, to which most people entrain (London, 2004). Accents can be created in numerous ways; among these, Lerdahl and Jackendoff identify three essential categories: phenomenal, structural, and metrical. Phenomenal accents include any attack points of pitch events, stresses caused by changes in articulation, dynamics, timbre, register, harmony, and so on. Structural accents are created by moments of "gravity" in tonal motion, particularly cadences. Lastly, metrical accents are beats that are made strong by their placement in a metrical context (Lerdahl \& Jackendoff, 1983, p. 17). Meter is created by regularly occurring patterns of phenomenal and structural accents that emphasize these beats; when enough of these accents align, a metrical context is made clear, and metrical accents emerge. In a simple quadruple meter (such as that which is often notated as 4/4), beats 1 and 3 are strong beats, whereas beats 2 and 4 are weak beats. Beat 1 is considered to be stronger than beat 3 , and therefore demarcates the 
beginning of a measure. This nested organization of strong and weak beats is referred to as the metrical hierarchy (Lerdahl \& Jackendoff, 1983).

Most rock songs (and songs under the broader umbrella of popular music) are in simple quadruple meter, which is made clear not by the main melodic layers (voice and guitar), but rather in the "overtly iterated accompaniment pattern" performed on the drums (Biamonte, 2014). So, while we acknowledge that all of the layers in a rock song will contribute to a listener's overall sense of meter and tempo, our study focuses entirely on the drums, as they contribute the most to these aspects of the music. In particular, we focus on the backbeat, considered by many to be a "foundational metric unit" in rock music (Biamonte, 2014). Simply put, the backbeat is the periodic accent of beats 2 and 4 of a measure in a simple quadruple meter. These accents are typically performed on the snare drum, and are an integral part of the standard rock drum beat, shown in Figure 1. Including the backbeat within standard theoretical models of meter, such as that presented by Lerdahl \& Jackendoff, is problematic. Because beats 2 and 4 are weak beats when compared to beats 1 and 3, the backbeat cannot be considered to be part of the metric hierarchy (See Figure 2). Instead, it creates a kind of syncopation: a temporary displacement of the regular, metric accent. This reduces backbeat to a local, stylistic rhythmic phenomenon. Yet, dismissing backbeat in this way is counterintuitive to its importance in the repertoire in which it is found. Kubik (2010, p. 45) refers to the backbeat as "the structural core of the musical piece" that enables "singers, drummers, and dancers ... to find their bearings." Further, several scholars argue that the backbeat coordinates the movements of musicians and listeners (Attas, 2014; Iyer, 2002; Kozak, 2018; Zbikowski, 2004), a perspective supported by ethnographic interviews with musicians (Attas, 2014). With this in mind, scholars such as Attas (2014), Butterfield (2006), and Zbikowski (2004) have each adapted various theories to account 
for backbeat as a metric phenomenon, rather than a rhythmic one. Attas, in particular, adapts London's (2004) metric theory to account for two "attentional peaks" in a simple quadruple meter: one reflecting the downbeat, and one reflecting the backbeat.

\section{Half Time and Double Time}

As described above, when the number of backbeats is suddenly halved, musicians often refer to the moment as "half time"; presumably, because the cycle of backbeats to which they are attending is transpiring at half the established rate. In Attas' terms, the backbeat cycle is now twice as long as the downbeat cycle. Musically, this effect can be easily created by the drummer by switching the snare accents from beats 2 and 4 of a measure to a single accent on beat 3

(Figure 3). Because the backbeat requires two snare attacks, the cycle is not completed until beat 3 of the following measure, effectively making the backbeat cycle last for two measures.

Because the backbeat is an essential, coordinating event within the music, the listener shifts their attention to the slower cycle, making the overall sense of tempo seem half as fast (i.e., half-time). Conversely, when the snare attacks double in rate, the listener would experience the music as transpiring twice as fast (i.e., double-time).

\section{Backbeat and Tempo Perception}

Although tactus is considered by many to correspond with listeners' perceptions of tempo (Drake et al., 2000), equating tactus rate to tempo may ignore other musical parameters, such as backbeat, that impact our sense of "musical speed" (Epstein, 1995). Is it possible that stylespecific parameters might have such an impact? Listeners are keenly aware of stylistic traits that define musical genres (Gjerdingen \& Perrott, 2008), and this has been shown to impact listeners' expectations of harmony (Vuvan \& Hughes, 2019), tempo, and meter (McKinney \& Moelants, 
2006). If the backbeat cycle is something to which most listeners entrain, it follows that any change in the rate of that cycle, such as the case of half or double time, could affect the listeners' overall perception of tempo. Since music analysts have long argued that backbeat can govern tempo modulations (Kozak, 2018), it would behoove us to test this theory in an experimental setting.

Two experiments were conducted to assess the effect of backbeat on tempo perception. In Experiment 1, listeners were presented with trials that combined shifts of BPM and backbeat. Experiment 2 was designed to isolate the effect of backbeat, and presented listeners with trials in which only backbeat shifted. We predicted in both experiments that shifts to double-time would lead participants to perceive the tempo as increasing, and that shifts to half-time would lead participants to perceive the tempo as decreasing.

\section{Experiment 1}

This study investigated the impact of backbeat on participants' accuracy in a tempo comparison task. Participants were asked to compare the tempos of pairs of excerpts, and judge whether the second excerpt of the pair was slower, the same tempo, or faster than the first. Excerpts were either 120 or 132 BPM, and pairs were combined to create either half-time, double-time, or consistent backbeat conditions. We predicted that a shift to higher BPM would lead to the perception of faster tempo, whereas a shift to lower BPM would lead to the perception of slower tempo. Likewise, we predicted that a backbeat shift to double-time would lead to the perception of faster tempo, whereas a backbeat shift to half-time would lead to the perception of slower tempo. Given that this is the first behavioural study, to our knowledge, to directly assess the concurrent effect of BPM and backbeat on tempo perception, we did not have specific 
predictions regarding how BPM and backbeat might affect one another. Thus we treated the congruent and incongruent interactions of these two variables as questions for exploration.

\section{Methods}

\section{Participants}

Participants $(N=21)$ were recruited from undergraduate classes for music majors and non-music majors at the [Institution redacted]. All participants were compensated for their participation with course credit. Gender and age data for the participants were not recorded. 18 reported formal musical training, with a mean duration of 10 years $(S D=8.84) .12$ participants reported that they currently play music, with a mean time spent per week of 12.25 hours $(S D=$ 6.54).

\section{Material}

All stimuli can be downloaded at https://osf.io/ecvr6/?view_only=65d014eb54f24e679fb635b43a853260. Four different songs, varying in melody and harmony, were composed to convey the conventions of rock music. These songs were played by a three-piece rock band consisting of electric guitar, bass, and drum set, and were 16 pulses in length ( 4 measures of 4 pulses each). To create the experimental stimuli, songs were manipulated in terms of BPM $(120,132)$ and backbeat. One backbeat consisted of a snare attack on either the second and fourth pulse of each measure ( $2 \& 4$; Figure 2$)$, and the other backbeat consisted of a snare attack on the third pulse of each measure (3; Figure 3).

To create experimental trials, songs were paired in sequence. The paired sequences always consisted of two identical songs, but could vary in BPM and backbeat. Critically, this 
pairing created four possible BPM shifts and four possible backbeat shifts within a trial. BPM could be consistent at $120 \mathrm{bpm}$, consistent at $132 \mathrm{bpm}$, faster (120-132 bpm), or slower (132-120 bpm). Backbeat could be consistent at 3, consistent at $2 \& 4$, double-time (3-2\&4), or half-time (2\&4-3). The combination of four BPM shifts and four backbeat shifts created 16 conditions for each of the four songs, thus producing 64 unique trials (see Table 1).

The experiment was presented on a Samsung Galaxy A tablet running OpenSesame 3.1 Runtime (Mathôt, Schreij, \& Theeuwes, 2012), and responses were collected via touchscreen. Participants listened to the stimuli using closed-back headphones.

\section{Procedure}

At the beginning of the experiment, participants were instructed with the following text:

"Thank you for participating in this music cognition experiment. In this study, we are interested in your impression of how fast music sounds. Each experiment trial features two excerpts heard one after another. Your job is to tell us whether the SECOND excerpt feels faster than the FIRST. Since we want your first impression, try to answer as quickly as possible.

Since we are interested in how fast it "feels" to you, don't conduct, tap, or bob your head to the music."

Then, six practice trials (randomly drawn from the pool of experimental trials) were presented to familiarize participants with the experiment. Finally, the 64 experimental trials were presented in random order. Each trial consisted of two 7-second excerpts, separated by 450ms of silence. Participants were able to respond at their own pace, though they were instructed to 
respond quickly with their first instinct. The experiment took approximately 17 minutes to complete.

\section{Results}

\section{Description of Participant Responses}

Table 2 shows the 16 different conditions of Experiment 1, organized into five categories that describe the ways in which BPM and backbeat combine: (1) Trials in which the BPM and backbeat of the two excerpts were consistent, (2) Trials in which only BPM changed, (3) Trials in which only backbeat changed, (4) Trials in which BPM and backbeat change congruently, and (5) Trials in which BPM and backbeat change incongruently. The last three columns of the table report the number of trials, aggregated across participants, on which participants responded that the second excerpt was faster, same, and slower than the first.

The pattern of responses for trials in which the BPM and backbeat were consistent is straightforward. Specifically, participants correctly report "same" to the majority of these trials (74\%). Perusing responses for trials in which only the BPM shifted, indicates that, as would be reasonably expected, BPM is a very strong cue for tempo. On trials in which the BPM increases from 120 to $132 \mathrm{bpm}$, participants report "faster" for $96 \%$ of trials. Conversely on trials in which the BPM decreases from 132 to $120 \mathrm{bpm}$, participants report "slower" for $95 \%$ of trials.

Our primary hypothesis concerned trials in which BPM was consistent while the backbeat shifted. In trials where the backbeat shifted to double-time, $48 \%$ of participant responses were "faster", $47 \%$ were "same", and only $5 \%$ were "slower". Thus, although backbeat does not seem to be as strong a cue for tempo as is BPM, double-time does seem to induce a feeling of 
increased tempo in participants. This effect is not as apparent for the half-time shift, with the majority of responses in this case (60\%) being "same", and only slightly more of the remaining responses being "slower" (27\%) than "faster" (14\%).

Finally, we explored the interactive effects of BPM and backbeat on tempo by looking at the conditions in which the BPM and backbeat shifted simultaneously. These data supported our previous observation that BPM is a stronger cue for tempo than is backbeat. Specifically, regardless of whether the BPM shift was congruent or incongruent with the backbeat shift, participants overwhelmingly responded in line with the direction of the BPM shift.

\section{Experiment 2}

In the second experiment, we aimed to observe as closely as possible the effect of backbeat by controlling for tempo variation, and focusing on trials where the backbeat changed (i.e., double-time vs. half-time). Thus, the experiment asked participants to compare the tempos of pairs of excerpts with the same tactus rate in a forced choice paradigm that required a response of either faster or slower (although the tempo was always the same). This provided us with a dependent variable that was more amenable to inferential statistics, as well as targeting our primary hypothesis with respect to backbeat. We predicted that backbeat shifts would affect tempo judgments, which would be reflected by the proportion of "slower" / "faster" responses being significantly different from chance (represented by a 50\% slower, 50\% faster distribution). Furthermore, we predicted that shifts to double-time would lead participants to judge the second excerpt as faster than the first, which would be shown by the proportion of "faster" responses being significantly higher than $50 \%$ in this condition. Finally, we predicted that shifts to halftime would lead participants to judge the second excerpt as slower than the first, which would be 
shown by the proportion of "slower" responses being significantly higher than $50 \%$ in this condition.

\section{Methods}

\section{Participants}

Participants were recruited from undergraduate classes for music majors and non-music majors. 28 participants identified as female, 16 identified as male. Mean age of participants was 21.70 years $(S D=2.83)$. Of the 44 participants, 34 identified as receiving some kind of musical training, with a mean duration of 10.85 years $(S D=5.87) .26$ participants reported that they currently play music, with a mean duration of 7.15 hours per week $(S D=3.23)$.

\section{Material}

All stimuli can be downloaded at https://osf.io/ecvr6/?view_only=65d014eb54f24e679fb635b43a853260. As in Experiment 1, four songs were composed for Experiment 2. Each song was played by a three-piece rock band consisting of electric guitar, bass, and drum set, and were 16 pulses in length (4 measures of 4 pulses each) . The songs were manipulated by BPM $(112,120,128,132)$, and backbeat $(2 \& 4,3)$. To create experimental trials, songs were paired in sequence. The paired sequences always consisted of two identical songs at two identical BPM. The backbeat shifted in every sequence, either from 3 to $2 \& 4$ (double-time) or from $2 \& 4$ to 3 (half-time). The combination of four songs, four BPMs, and two backbeat shifts created 32 unique trials, each of which was presented twice, producing a final set of 64 experimental trials. 
The experiment was presented on a Samsung Galaxy A tablet running OpenSesame 3.1 Runtime (Mathôt, Schreij, \& Theeuwes, 2012), and responses were collected via touchscreen. Participants listened to the stimuli using closed-back headphones.

\section{Procedure}

Participants received the same instructions as in Experiment 1, except that they were only able to respond "faster" or "slower" ("same" was no longer an option). Six practice trials (randomly drawn from the pool of experimental trials) were presented to familiarize participants with the experiment. Then, the 64 experimental trials were presented in random order. Each trial consisted of the two excerpts, separated by $450 \mathrm{~ms}$ of silence. Participants were able to respond at their own pace, though they were instructed to respond quickly with their first instinct. The experiment took approximately 22 minutes to complete.

\section{Results}

Participant responses were collapsed across song, tempo, and repetition, as these variables were not of interest, and did not vary within trials. For the Double-Time condition, a one-sample t-test compared the proportion of 'faster' responses to a value of 16 (50\% of double-time trials), which would indicate chance performance. There was a significantly larger proportion of 'faster' responses than would be expected by chance, $M=24.66,95 \%$ CI $[23.37,25.95], t(43)=$ $13.52, p<.001$. For the Half-Time condition, a one-sample t-test compared the proportion of 'faster' responses to a value of 16 (50\% of half-time trials), which would indicate chance performance. There was a significantly lower proportion of 'faster' responses (i.e., higher proportion of 'slower' responses) than would be expected by chance, $M=12.11,95 \% \mathrm{CI}$ $[10.41,13.82], t(43)=4.60, p<.001$. A paired-samples t-test compared the proportions of 
'faster' responses in the double-time and half-time conditions and showed the proportions of these responses to be significantly different from one another, $M_{d}=12.55,95 \%$ CI $[9.85$, 15.24], $t(43)=9.39, p<.001$. These results are summarized in Figure 4.

Our last analysis assessed the effect of years of musical training on the degree to which backbeat affects participant responses. To this end, we extracted the proportion of "faster" responses for each backbeat condition (Double-Time, Half-Time), calculated the absolute deviation from chance (0.50), and then averaged this deviation over the two conditions. This deviation measure quantified for each participant the strength of the effect of backbeat on their tempo judgments, with higher deviations indicating a larger effect of backbeat. Finally, participants' deviation scores were correlated with their number of years of musical training. There was no significant correlation, $r(42)=-.11, p=.49$, indicating that the effect of backbeat on tempo perception was not affected by musical training.

\section{Discussion}

The results of both experiments confirm our hypotheses that backbeat affects listeners' perception of tempo. More specifically, when the backbeat shifts to create a double-time or halftime feel, listeners will perceive the tempo to be faster or slower, respectively. This becomes most clear when the tactus stays the same while the backbeat changes, as in Experiment 2.

In addition to salient cues to tempo such as BPM, listeners may attend to the dynamic accent patterns created by the backbeat when constructing their perceived metrical hierarchy. Following the work of Drake et al. (1999), our results suggest that the manipulation of the backbeat in ecologically valid stimuli such as the ones used in our experiments will create a distinction between physical and perceived tempo. One explanation for this may derive from 
Jones \& Boltz's dynamic attending theory (1989), which would suggest that, upon establishing an initial perceived tempo based on the tactus at the onset of the stimulus, listeners then shift their attention to the backbeat cycle. If the backbeat cycles differ between two compared stimuli, listeners will report this difference as a change in tempo. A competing explanation for our results could be that stimuli with different backbeat cycles produce different event densities.

Comparison of stimuli with different event densities would result in the perception of a difference in tempo. A double-time backbeat cycle shift would have a higher event density, and therefore be perceived as faster, while a half-time backbeat cycle shift would have a lower event density, and would be perceived as a slowing down of the tempo. This explanation would align with the results reported by Bisesi \& Vicario (2015) and Drake et al. (1999). Of these two competing explanations, we prefer dynamic attending theory, as research on the impact of event density on tempo perception suggests that event density has its largest impact when the stimuli consist of non-isochronous or random sequences. Our stimuli consisted of ecologically-valid, entrainable music with prominent, consistent backbeat cycles. Though more research on this is needed, we posit that, following Attas (2014), the backbeat presents a second attentional peak that draws in the listener once the tactus is established. Like the findings of Boltz (2011) and London (2011), our results suggest that tactus does not necessarily equate with tempo, and that non-tactus and factors can have a significant impact on its perception.

Numerous attempts at automatic tempo extraction (Klapuri et al., 2006) have revealed what Moelants (2002) calls a "tempo-octave" error - an error in which tempo is assigned to a metrical level once removed from the detected tactus in the hierarchy. This error aligns precisely with the difference highlighted by our study. If backbeat, rather than tactus, helps to define the 
metrical hierarchy in popular music, then tempo extraction algorithms may benefit from assigning it more weight.

Although our experiments were not designed to directly address the perception of the metrical hierarchy, we would be remiss if we failed to acknowledge the impact of our study on the apparent impasse between traditional metric theory and the seemingly vital role that backbeat serves in the musical styles in which it is commonplace. Lerdahl and Jackendoff's (1983) theory would explain backbeat as a surface-level, rhythmic phenomenon; yet, many scholars have argued that backbeat serves as a governing, orienting force in specific repertories (Attas, 2014; Biamonte, 2014; Butterfield, 2006; Iyer, 2002; Kozak, 2018; Kubik, 2010). Our results suggest that, in style-specific conditions such as those presented in our experiments, backbeat is a musical feature to which listeners readily attend. Given this, it would seem plausible that backbeat could serve a greater role in the formation of our mental representations of a stylespecific metric hierarchy than Lerdahl and Jackendoff's theory would imply. Some scholars have adapted Lerdahl and Jackendoff's theory to other musical styles and contexts (London, 2004; Temperley, 2010); this is something that the results of our study would support, though the scope of redesigning that theory goes beyond the scope of this article. Both van Noorden and Moelants (1999) and McKinney and Moelants (2006) show that tempo perception varies as a function of musical style; these results are further confirmed by the present study. Similarly, Trevor de Clercq (2016) argues that measures (and by extension, the metrical hierarchy) in popular music are defined by durational boundaries of approximately 2 seconds. While de Clercq's argument stems from a perspective of music analysis rather than perception, it nevertheless supports the notion that representations of the metrical hierarchy should be style-specific. More generally, our findings suggest that listeners are keenly aware of musical style, and that style should thus be an 
important factor for general theories of music cognition; a claim further supported by a growing body of research (Gjerdingen \& Perrott, 2008; Vuvan \& Hughes, 2019).

\section{Future Research}

Previous research on tempo perception has suggested that musicians tended to entrain to a slower tactus than non-musicians (Drake et al., 1999). Musical training did not impact our results in either of our experiments, which contradicts these findings. However, given that our experiments were not specifically designed to address musical training, these analyses were underpowered. Further investigation into the effect of training on backbeat-shift-influenced tempo perception would require a larger sample recruited specifically to address the issue of varying experience with musical training.

Both of our experiments used a post-hoc, forced-choice framework that may have created an unrealistic musical environment for participants. A follow-up study using a response mode that avoids the direct comparison of pairs of excerpts may offer further insight into the notion of "how fast music feels." Similarly, it would behoove us to design follow-up studies that utilize tapping paradigms so that our results could compare more directly with previous research (Drake et al., 2000; P. A. Martens, 2011; McKinney \& Moelants, 2006; Snyder \& Krumhansl, 2001). A study that uses implicit non-behavioural responses as an index for perceived tempo, such as neural tagging using electroencephalography (e.g., Nozaradan, Peretz, Missal, \& Mouraux, 2011) would potentially address possible confounds, such as kinematic constraints or fatigue due to a design that requires an explicit participant response. Finally, our experiments only investigated the impact of backbeat on tempo perception, without regard for the connection between BPM and backbeat. It would be beneficial to further investigate how these two parameters might interact in determining perceived tempo. 


\section{References}

Attas, R. (2014). Meter and Motion in Pop/Rock Backbeats. Society for Music Theory, Milwaukee, Wisconsin.

Biamonte, N. (2014). Formal Functions of Metric Dissonance in Rock Music. Music Theory Online, 20(2). http://www.mtosmt.org/issues/mto.14.20.2/mto.14.20.2.biamonte.html

Bisesi, E., \& Vicario, G. B. (2015). The Perception of an Optimal Tempo: The Role of Melodic Events Density. In A. Galmonte \& R. Actis-Grosso (Eds.), Different Psychological Perspectives on Cognitive Processes: Current Research Trends in Alps-Adria Region (pp. 25-43). Cambridge Scholars Publishing.

Boltz, M. G. (2011). Illusory Tempo Changes Due to Musical Characteristics. Music Perception, 28(4), 367-386. https://doi.org/10.1525/mp.2011.28.4.367

Butterfield, M. W. (2006). The Power of Anacrusis: Engendered Feeling in Groove-Based Musics. Music Theory Online, 12(4). http://www.mtosmt.org/issues/mto.06.12.4/mto.06.12.4.butterfield.html

Danielsen, A. (Ed.). (2010). Musical rhythm in the age of digital reproduction. Ashgate. de Clercq, T. (2016). Measuring a Measure: Absolute Time as a Factor for Determining Bar Lengths and Meter in Pop/Rock Music. Music Theory Online, 22(3). http://mtosmt.org/issues/mto.16.22.3/mto.16.22.3.declercq.html

Drake, C., \& Botte, M.-C. (1993). Tempo sensitivity in auditory sequences: Evidence for a multiple-look model. Perception \& Psychophysics, 54(3), 277-286. https://doi.org/10.3758/BF03205262

Drake, C., Gros, L., \& Penel, A. (1999). How fast is that music? The relation between physical and perceived tempo. Music, Mind, and Science, 190-203. 
Drake, C., Penel, A., \& Bigand, E. (2000). Tapping in Time with Mechanically and Expressively Performed Music. Music Perception, 18(1), 1-23. https://doi.org/10.2307/40285899

Elowsson, A., \& Friberg, A. (2013). Modelling Perception of Speed in Music Audio.

Proceedings of the Sound and Music Computing Conference 2013,. Sound and Music Computing, Stockholm, Sweden.

http://citeseerx.ist.psu.edu/viewdoc/summary?doi=10.1.1.416.7300

Epstein, D. (1995). Shaping time: Music, the brain, and performance. Schirmer Books.

Gjerdingen, R. O., \& Perrott, D. (2008). Scanning the Dial: The Rapid Recognition of Music Genres. Journal of New Music Research, 37(2), 93-100. https://doi.org/10.1080/09298210802479268

Honing, H. (2013). Structure and Interpretation of Rhythm in Music. In The Psychology of Music (pp. 369-404).

Iyer, V. (2002). Embodied Mind, Situated Cognition, and Expressive Microtiming in AfricanAmerican Music. Music Perception, 19(3), 387-414. https://doi.org/10.1525/mp.2002.19.3.387

Jones, M. R., \& Boltz, M. (1989). Dynamic attending and responses to time. Psychol. Rev., 96(3), 459-491. https://doi.org/10.1037/0033-295X.96.3.459

Kozak, M. (2018). “Headbanging to 'Giant Steps': Backbeat and Tempo Modulation in the Music of Panzerballett." Society for Music Theory, San Antonio, TX.

Kubik, G. (2010). Theory of African Music (Vol. 1). University of Chicago Press. https://www.scribd.com/book/380644710/Theory-of-African-Music-Volume-I

Lerdahl, F., \& Jackendoff, R. (1983). A generative theory of tonal music. MIT Press.

London, J. (2004). Hearing in time: Psychological aspects of musical meter. Oxford University Press. 
London, J. (2011). Tactus $\neq$ Tempo: Some Dissociations Between Attentional Focus, Motor Behavior, and Tempo Judgment. Empir. Musicol. Rev., 6(1), 43-55. https://doi.org/10.18061/1811/49761

Martens, P. A. (2011). The Ambiguous Tactus: Tempo, Subdivision Benefit, And Three Listener Strategies. Music Perception, 28(5), 433-448. https://doi.org/10.1525/mp.2011.28.5.433

Martens, P., \& Benadon, F. (2017). Musical Structure: Time and Rhythm. Routledge Handbooks Online. https://doi.org/10.4324/9781315194738.ch10

McKinney, M. F., \& Moelants, D. (2006). Ambiguity in Tempo Perception: What Draws Listeners to Different Metrical Levels? Music Perception, 24(2), 155-166. https://doi.org/10.1525/mp.2006.24.2.155

Moelants, D. (2006). Perception and performance of aksak metres. Music Sci., 10(2), 147-172. https://doi.org/10.1177/102986490601000201

Nozaradan, S., Peretz, I., Missal, M., \& Mouraux, A. (2011). Tagging the neuronal entrainment to beat and meter. J. Neurosci., 31(28), 10234-10240. https://doi.org/10.1523/JNEUROSCI.0411-11.2011

Parncutt, R. (1994). A Perceptual Model of Pulse Salience and Metrical Accent in Musical Rhythms. Music Perception, 11(4), 409-464. https://doi.org/10.2307/40285633

Quinn, S., \& Watt, R. (2006). The Perception of Tempo in Music. Perception, 35(2), 267-280. https://doi.org/10.1068/p5353

Snyder, J., \& Krumhansl, C. L. (2001). Tapping to Ragtime: Cues to Pulse Finding. Music Perception, 18(4), 455-489. https://doi.org/10.1525/mp.2001.18.4.455

Temperley, D. (2010). Modeling Common-Practice Rhythm. Music Perception: An Interdisciplinary Journal, 27(5), 355-376. https://doi.org/10.1525/mp.2010.27.5.355 van Noorden, L., \& Moelants, D. (1999). Resonance in the Perception of Musical Pulse. Journal 
of New Music Research, 28(1), 43-66. https://doi.org/10.1076/jnmr.28.1.43.3122

Vuvan, D. T., \& Hughes, B. (2019). Musical Style Affects the Strength of Harmonic Expectancy. Music \& Science, 1(1). https://doi.org/10.1177/2059204318816066

Zbikowski, L. M. (2004). Modelling the Groove: Conceptual Structure and Popular Music. Journal of the Royal Musical Association, 129(2), 272-297. 


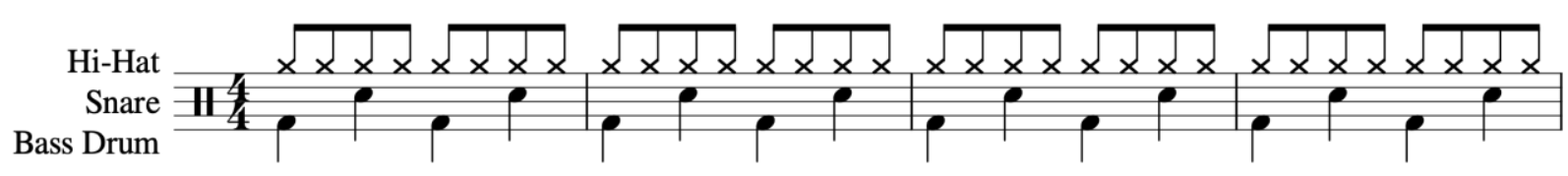

Figure 1. A standard rock drum beat. The backbeat occurs on beats 2 and 4 of each measure, played on the snare drum. 


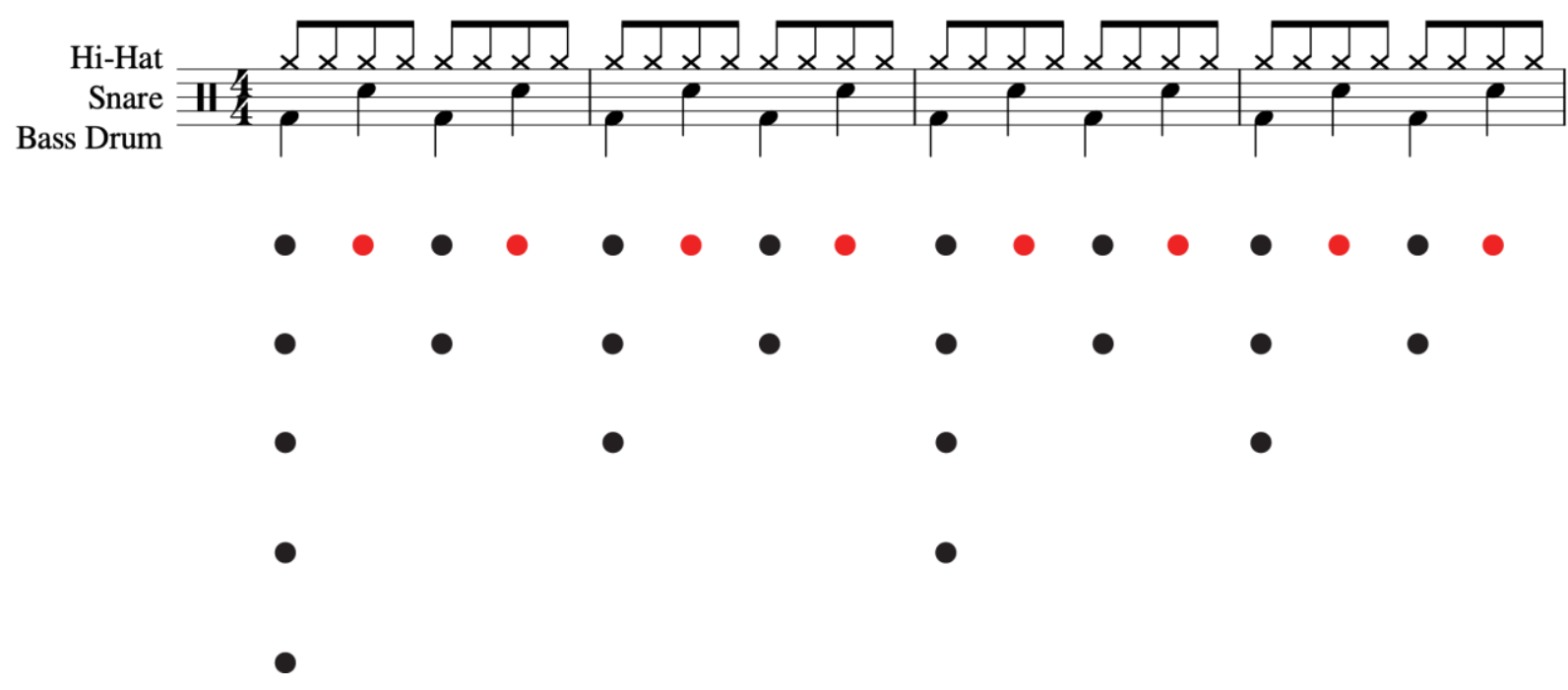

Figure 2. A metric analysis of the standard rock drum beat, using the methodology from Lerdahl \& Jackendoff (1983). The backbeat, indicated by the red dots in the metric analysis, does not extend beyond the beat level because it is not a strong beat within the measure. It is erased at the half-measure level, suggesting that it does not form part of the underlying metrical hierarchy.

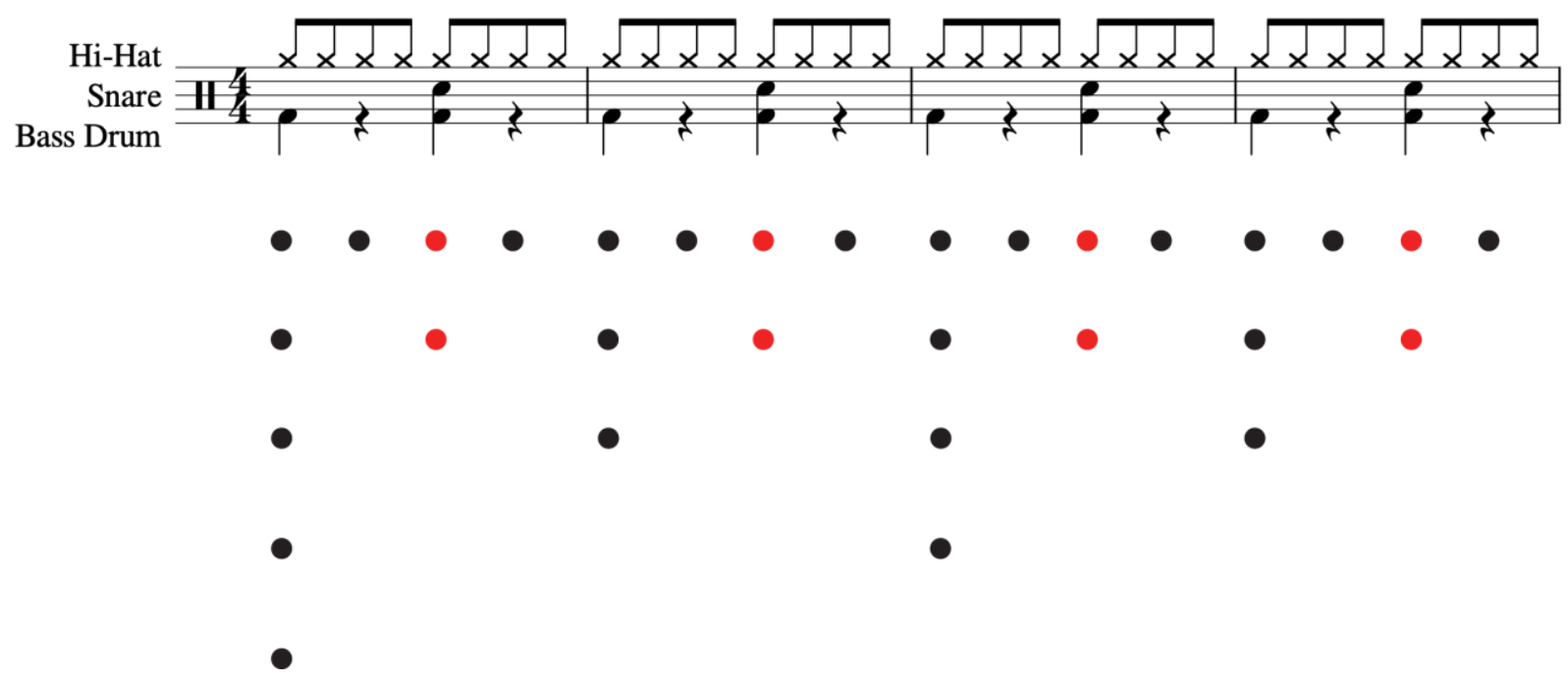

Figure 3. A metric analysis of a half-time rock drum beat. The backbeat, indicated by the red 
dots, now aligns with the strong third beat of the measure, and is extended to the half-measure level of the metric hierarchy. The backbeat is still erased by the strength of the downbeat cycle. The cycle of backbeat attacks now takes twice as long to complete as it did in Figure 2. 


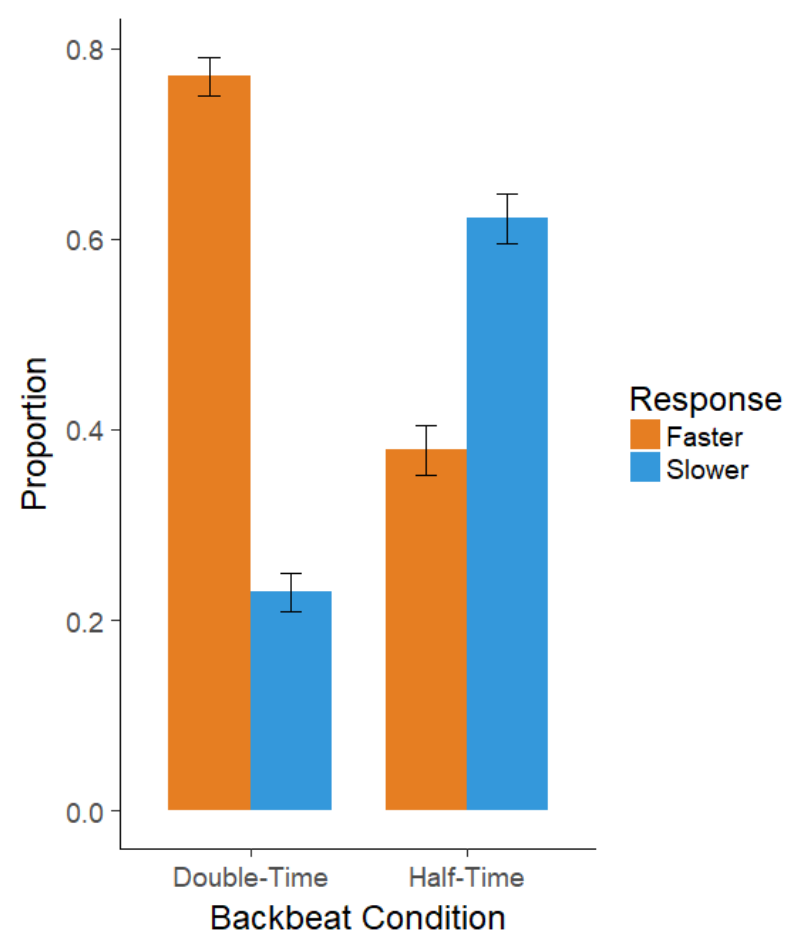

Figure 4. Proportion of responses in each of the Backbeat conditions (half-time and double-time) in Experiment 2. Error bars represent standard error of the mean. 
Table 1

All 16 tempo and snare conditions for Experiment 1. Each condition was used in 4 different song contexts, for a total of 64 trials. Trials were presented in a random order.

\begin{tabular}{|c|c|c|c|}
\hline Tempo 1 & Snare 1 & Tempo 2 & Snare 2 \\
\hline 120 & $2 \& 4$ & 120 & $2 \& 4$ \\
\hline 120 & $2 \& 4$ & 132 & $2 \& 4$ \\
\hline 120 & 3 & 120 & 3 \\
\hline 120 & 3 & 132 & 3 \\
\hline 120 & $2 \& 4$ & 120 & 3 \\
\hline 120 & $2 \& 4$ & 132 & 3 \\
\hline 120 & 3 & 120 & $2 \& 4$ \\
\hline 120 & 3 & 132 & $2 \& 4$ \\
\hline 132 & $2 \& 4$ & 120 & $2 \& 4$ \\
\hline 132 & $2 \& 4$ & 132 & $2 \& 4$ \\
\hline 132 & 3 & 120 & 3 \\
\hline 132 & 3 & 132 & 3 \\
\hline 132 & $2 \& 4$ & 120 & 3 \\
\hline 132 & $2 \& 4$ & 132 & 3 \\
\hline 132 & 3 & 120 & $2 \& 4$ \\
\hline 132 & 3 & 132 & $2 \& 4$ \\
\hline
\end{tabular}


Table 2

Percentage of "Faster", "Same”, and "Slower" Responses in Experiment 1

\begin{tabular}{|c|c|c|c|c|c|c|c|c|c|}
\hline Change & BPM 1 & BPM 2 & $\begin{array}{c}\text { Backbeat } \\
1\end{array}$ & $\begin{array}{c}\text { Backbeat } \\
2 \\
\end{array}$ & $\begin{array}{c}\text { BPM } \\
\text { Change }\end{array}$ & $\begin{array}{l}\text { Backbeat } \\
\text { Change }\end{array}$ & $\begin{array}{c}\mathrm{N} \\
\text { Faster }\end{array}$ & $\begin{array}{c}\mathrm{N} \\
\text { Same }\end{array}$ & $\begin{array}{c}\mathrm{N} \\
\text { Slower }\end{array}$ \\
\hline \multicolumn{10}{|l|}{ BPM / Backbeat } \\
\hline \multirow[t]{5}{*}{ Consistent } & 120 & 120 & $2 \& 4$ & $2 \& 4$ & Consistent & Consistent & 12 & 64 & 8 \\
\hline & 120 & 120 & 3 & 3 & Consistent & Consistent & 8 & 65 & 11 \\
\hline & 132 & 132 & $2 \& 4$ & $2 \& 4$ & Consistent & Consistent & 24 & 60 & 0 \\
\hline & 132 & 132 & 3 & 3 & Consistent & Consistent & 21 & 61 & 2 \\
\hline & & & & & & & $19 \%$ & $74 \%$ & $6 \%$ \\
\hline \multicolumn{10}{|l|}{ BPM Change } \\
\hline \multirow[t]{6}{*}{ Only } & 120 & 132 & $2 \& 4$ & $2 \& 4$ & Increase & Consistent & 81 & 3 & 0 \\
\hline & 120 & 132 & 3 & 3 & Increase & Consistent & 81 & 3 & 0 \\
\hline & & & & & & & $96 \%$ & $4 \%$ & $0 \%$ \\
\hline & 132 & 120 & $2 \& 4$ & $2 \& 4$ & Decrease & Consistent & 0 & 5 & 79 \\
\hline & 132 & 120 & 3 & 3 & Decrease & Consistent & 0 & 4 & 80 \\
\hline & & & & & & & $0 \%$ & $5 \%$ & $95 \%$ \\
\hline \multirow{7}{*}{$\begin{array}{l}\text { Backbeat } \\
\text { Change Only }\end{array}$} & & & & & & & & & \\
\hline & 120 & 120 & 3 & $2 \& 4$ & Consistent & Double Time & 31 & 45 & 8 \\
\hline & 132 & 132 & 3 & $2 \& 4$ & Consistent & Double Time & 49 & 34 & 1 \\
\hline & & & & & & & $48 \%$ & $47 \%$ & $5 \%$ \\
\hline & 120 & 120 & $2 \& 4$ & 3 & Consistent & Half Time & 6 & 48 & 30 \\
\hline & 132 & 132 & $2 \& 4$ & 3 & Consistent & Half Time & 17 & 52 & 15 \\
\hline & & & & & & & $14 \%$ & $60 \%$ & $27 \%$ \\
\hline \multirow[t]{4}{*}{$\begin{array}{l}\text { BPM / Backbeat } \\
\text { Congruent }\end{array}$} & 120 & 132 & 3 & $2 \& 4$ & Increase & Double Time & 83 & 1 & 0 \\
\hline & & & & & & & $99 \%$ & $1 \%$ & $0 \%$ \\
\hline & 132 & 120 & $2 \& 4$ & 3 & Decrease & Half Time & 1 & 3 & 80 \\
\hline & & & & & & & $1 \%$ & $4 \%$ & $95 \%$ \\
\hline \multirow[t]{4}{*}{$\begin{array}{l}\text { BPM / Backbeat } \\
\text { Incongruent }\end{array}$} & 120 & 132 & $2 \& 4$ & 3 & Increase & Half Time & 76 & 7 & 1 \\
\hline & & & & & & & $90 \%$ & $8 \%$ & $1 \%$ \\
\hline & 132 & 120 & 3 & $2 \& 4$ & Decrease & Double Time & 6 & 9 & 69 \\
\hline & & & & & & & $7 \%$ & $11 \%$ & $82 \%$ \\
\hline
\end{tabular}

